\title{
MULTI-OBJECTIVE CONTROL WITH MODAL NOTCHING VIA IMSC
}

\author{
Smith, M.J. and Berg, J.L. * \\ USAF Phillips Laboratory \\ Edwards AFB, CA 93523-5000
}

June 10, 1992

\begin{abstract}
This paper develops a control methodology that allocates control authority for specific modes of a linear system via the IMSC technique while projecting excess control authority onto an orthogonal space which independently satisfies other control nbjectives. The orthogonal control authority used in this paper is LQG-based, and the resulting control system turns out to be the solution of an output feedback problem.
\end{abstract}

\section{Introduction}

Future spacecraft such as the envisioned Space-Based Radar (SBR) will be composed of several substructures which can be classified as Large Space Structures (LSS). Examples of LSS include reflector antennas, radar membranes, radar arrays and solar arrays. It's common to have LSS at tached to orbiting vehicles (e.g. the satellites deployed by the National Geological Survey).

Orbiting vehicles are commonly exposed to internal excitation such as the onboard rotating machinary mentioned in reference to cryogenic coolers [1], as well as exogenious disturbances due to slewing, temperature gradients, pulse excitations, etc. A control technique that distinguishes frequency dependent excitations from random disturbances and controls both using orthogonal control authority hernmes beneficial

"The authors wish to express their appreciation to the Air Force Office of Scientific Research for their - s r throughout this research. under such conditions. This paper develops a control scheme that orthogonally notches out excitation from frequency dependent sources such as critical resonant modes and simultaneously dampens the excitation from random disturbances. Control simulations using this technique are shown to be convincing.

\section{IMSC Review}

It is important to review the IMSC control technique developed by Meirovitch in the early 80 's [2]. The IMSC technique seeks modal forces, which can be transformed to the configuration space to acquire actuator forces. When the number of independent actuators equals the number of controlled modes, a matrix inversion of the modal participation matrix provides the transformation to the configuration space, otherwise a pseudo-inversion is carried out which yields a coupled control law referred to as pseudo-inverse IMSC. The following derivation is a synopsis of the work done by Meirovitch, et al. ([3] through [5]).

The mathematical description of a flexible, viscously damped structure can be modelled in modal coordinates as:

$$
\begin{gathered}
\ddot{\vec{\eta}}(t)+\Xi \dot{\vec{\eta}}(t)+\Lambda \vec{\eta}(t)=\Phi^{T} B_{m} \vec{u}(t)+\Phi^{T} D_{m} \vec{w}(t) \\
\vec{y}(t) \doteq C\left\{\begin{array}{c}
\vec{\eta}(t) \\
\dot{\vec{\eta}}(t)
\end{array}\right\}
\end{gathered}
$$

where $\vec{\eta}(t)$ is the vector of modal amplitudes, $[\Xi]$ and $[\Lambda]$ are diagonal matrices which represent the system damping and stiffness, respectively $([\Xi]=$ $\left.\operatorname{diag}\left(2 \zeta_{r} \omega_{r}\right),[\Lambda]=\operatorname{diag}\left(\omega_{r}^{2}\right)\right) .[\Phi]$ is the modal matrix. $\vec{u}(t)$ is the control input, $\vec{y}(t)$ is the system's 
output and $\vec{w}(t)$ is the external disturbance. For con; ciseness, the following matrices defined as

$$
[B \quad D] \doteq \Phi^{T}\left[B_{m} D_{m}\right]
$$

will be used in the remaining portion of this paper.

Assuming the modal state is obtainable, we may choose diagonal gain matrices in order to maintain independence for the controlled modes. The modal control law becomes:

$$
\begin{aligned}
& B \vec{u}(t)=\left[G_{1} G_{2}\right]\left\{\begin{array}{c}
\hat{\eta}(t) \\
\dot{\hat{\eta}}(t)
\end{array}\right\} \\
& ; G_{1} \doteq \operatorname{diag}\left(g_{11} g_{12} \ldots g_{1 n}\right) \\
& ; G_{2} \doteq \operatorname{diag}\left(g_{21} g_{22} \ldots g_{2 n}\right)
\end{aligned}
$$

where $\hat{\eta}(t)$ and $\dot{\dot{\eta}}(t)$ represent the estimated modal position and velocity coordinates of the structure, provided by modal filters [6]. The control vector in the configuration space, $\vec{u}(t)$, can be uniquely determined, provided there are as many independent actuators as there are modes to be controlled.

$$
\vec{u}(t)=B^{-1}\left[G_{1} G_{2}\right]\left\{\begin{array}{l}
\dot{\eta}(t) \\
\dot{\hat{\eta}}(t)
\end{array}\right\}
$$

The existence of the true inverse of the transpose of the modal matrix permits the controller to maintain independence between the modes for the closed-loop system, thereby allowing one to control the modes individually. This yields higher computational efficiency during the solution of the Riccatti Equations, performed during the determination of the optimal gains.

Extended work in IMSC includes that of Sinha [7], in which a sliding mode approach to account for system nonlinearities has been proposed. Lindberg and Longman [8] studied the questions of actuator quantity and placement using the IMSC technique. An approach for using fewer actuators than the number of controlled modes without resorting to pseudoinversion is addressed by $\mathrm{Baz}$ and Poh [9]. Silverberg and Meirovitch [10] proposed a solution to dynamic limitations of actuators by targeting specified modes to be controlled by blocks of actuators. Nguyen [11] also addressed actuator issues using IMSC. More recently, an experimental comparison of IMSC and Linear Zuadratic control is performed by Berg [12], in which the pseudo-inverse IMSC controller was found to be more robust with respect to uncertainties in the system dynamics with the penalty of slightly higher energy requirements. It's also important to mention here that numerous papers have been published on partial and regional pole assignment with varying control objectives, and recent papers by Haddad and Bernatein [13] and Lu, etc. [14], contain a well documented list of sources.

\section{Main Result}

It is expected that space structures will experience excitation sources that are both frequency dependent and random. It turns out that IMSC can be used to dampen key resonant modes while simultaneously controlling, in an orthogonal manner, random disturbances. This allows one to control Line-of-Sight (LOS) errors by satisfying multiple control objectives. We begin by introducing the following theorem:

Theorem 1 When more independent actuators than designated modes are used in the IMSC method, multiple control objectives can be obtained by constraining excess control authority to the null space of the spacial rows of the modal forces.

Proof 1 lemma 1 (Rao and Mitra,[15]) The equation $L X R=Y$ can be solved for $X$ iff $L, R$, and $Y$ have the property $L L^{\prime} Y R^{\prime} R=Y$ where $L^{\prime}$ and $R^{\prime}$ are any matrices satisfying

$$
\begin{aligned}
L L^{\prime} L & =L \\
R R^{\prime} R & =R
\end{aligned}
$$

and if there exists any solutions, then all of them are given by

$$
X=L^{\prime} Y R^{\prime}-L^{\prime} L Z R R^{\prime}+Z
$$

where $Z$ is an arbitrary matrix of correct dimension.

Recall that the IMSC controller is given by Equation (3), but when more independent actuators are used than the number modes one wishes to control, application of the lemma to Equation (2), choosing $[L] \doteq[B]$ and $[R] \doteq[I]$, leaves us with the solution: ${ }^{\prime}$

$$
\vec{u}(t)=B^{+}\left[G_{1} G_{2}\right]\left\{\begin{array}{c}
\hat{\eta}(t) \\
\dot{\hat{\eta}}(t)
\end{array}\right\}+\left[I-B^{+} B\right] Z
$$

${ }^{1}[L]^{+} \doteq$ pseudo-inverse of $[L]$ 
where $[Z]$ is a free matrix of correct dimension. Substitution of

$$
Z=G_{z}\left\{\begin{array}{c}
\hat{\eta}(t) \\
\dot{\hat{\eta}}(t)
\end{array}\right\}
$$

into Equation (4) gives an alternate set of solutions

$$
\vec{u}(t)=\left[B^{+}\left[G_{1} G_{2}\right]+\left[I-B^{+} B\right] G_{z}\right]\left\{\begin{array}{c}
\dot{\eta}(t) \\
\dot{\dot{\eta}}(t)
\end{array}\right\}
$$

where the control gains $G_{1}$ and $G_{2}$ are devoted to the control of specific modes (modal notching) and $G_{z}$ provides freedom for additional control objectives.

\subsection{Comment}

One can see that minimizing the norm on the gain equates to minimizing the function:

$$
\begin{aligned}
f\left(G_{z}\right) & \doteq\left\|B^{+}\left[G_{1} G_{2}\right]+\left[I-B^{+} B\right] G_{z}\right\| \\
& \geq\left\|B^{+}\left[G_{1} G_{2}\right]\right\| \forall G_{z}
\end{aligned}
$$

This varifies our suspicion that any control objective beyond control of specific modes using the modal notching technique will result in additional control effort.

\section{Modal Notch Control with Gen- eralized Measurement}

In this section, we construct the first-order, state space form which contains the previously dedicated control for a subset of $r$ plant modes, and the remaining control authority will be allocated for various objectives.

\subsection{State Space Construction}

In flexible space structures, inertial positions, rates, and accelerations are typically provided by onboard instrumentation. The measurement vector is generalized as:

$$
\vec{z}(t)=H_{1} \vec{\eta}(t)+H_{2} \dot{\vec{\eta}}(t)+H_{3} \ddot{\vec{\eta}}(t)+\vec{\nu}(t)
$$

or equivalantly (by substitution of Equation (1))

$$
\begin{aligned}
\vec{z}(t)= & {\left[\left[H_{1} H_{2}\right]-H_{3}[\Lambda \Xi]\right]\left\{\begin{array}{c}
\vec{\eta}(t) \\
\dot{\vec{\eta}}(t)
\end{array}\right\} } \\
& +H_{3}\{B \vec{u}(t)+D \vec{w}(t)\}+\vec{\nu}(t)
\end{aligned}
$$

We define the state vector as:

$$
\vec{q} \doteq\left\{\begin{array}{l}
\vec{\eta}(t) \\
\dot{\eta}(t)
\end{array}\right\}
$$

and the matrices:

$$
\begin{aligned}
\hat{A} & \doteq\left[\begin{array}{cc}
0 & I \\
-\Lambda & -\Xi
\end{array}\right] \quad \hat{B} \doteq\left[\begin{array}{l}
0 \\
B
\end{array}\right] \dot{E} \doteq\left[\begin{array}{c}
0 \\
D
\end{array}\right] \\
\hat{C} & \doteq\left[\left[H_{1} H_{2}\right]-H_{3}[\Lambda \quad \Xi]\right] \\
\hat{D} & \doteq H_{3} B \quad \hat{F} \doteq H_{3} D
\end{aligned}
$$

The plant and measurement vectors are:

$$
\begin{aligned}
& \dot{q}(t)=\hat{A} \vec{q}(t)+\hat{B} \vec{u}(t)+\hat{E} \vec{w}(t) \\
& \vec{z}(t)=\hat{C} \vec{q}(t)+\hat{D} \vec{u}(t)+\hat{F} \vec{w}(t)+\vec{\nu}(t)
\end{aligned}
$$

with the estimated state $\hat{\eta}$, the modal notch controller is:

$$
\vec{u}(t)=\left[B_{r}^{+}\left[G_{1} G_{2}\right] H_{n}+\left[I-B_{r}^{+} B_{r}\right] G_{z}\right] \hat{q}(t) .
$$

where the matrix $B_{r} \doteq \Phi^{T}{ }_{r} B_{m}$ constrains $\mathrm{r}$ modes for the notch controller. It is clear from the previous development that $\mathrm{I}$ modes are guaranteed to have the damping characteristics by the IMSC methodology, and the remaining control freedom (gain matrix $\left[G_{z}\right]$ ) can be dedicated to other control purposes. The matrix $H_{m}$ is a rectangular matrix whose rows consist of standard unit vectors. $H_{m}$ is used to factor out the modes of interest for the IMSC controller from a more generalized set of modes. The larger set of modes provides a more observable system for the orthogonal controller (i.e., the LQG control in this paper).

Control objectives are problem dependent which makes the ability of the Modal Notch Control to accomodate various control schemes, applicable to many different practical problems. In this paper, we will show that important modes can be controlled while an LQG based control scheme simultaneously achieves LOS pointing accuracy for a flexible space structure. Simulations using this control technique are applied to the FEM model of ASTREX (a test facility at the Phillips Laboratory, Edwards AFB, CA) with the results presented in Section 6.

\section{Optimal Estimation \& Control with Modal Notching}

The issue of finding the optimal estimator with correlated plant and measurement noise has been resolved 
in [16] and has been used in an application concerning an actuator and sensor study [17]. The system described by the Equations (5) clearly has correlated noise, and the optimal estimator matrix can be found by solving a standard Riccatti equation as described below.

By defining the expected noise values:

$$
W \doteq E_{\infty}\left(\vec{w}(t) \vec{w}^{T}(t)\right) \quad V \doteq E_{\infty}\left(\vec{\nu}(t) \vec{\nu}^{T}(t)\right)
$$

and the matrix

$$
\left[\begin{array}{ll}
V_{11} & V_{12} \\
V_{21} & V_{22}
\end{array}\right] \doteq\left[\begin{array}{cc}
\hat{E} W \hat{E}^{T} & \hat{E} W \hat{F}^{T} \\
\hat{F} W \hat{E}^{T} & \hat{F} W \hat{F}^{T}+V
\end{array}\right]
$$

one can find the optimal estimator matrix $[L]$ by solving the Riccatti equation:

$$
\begin{aligned}
& X_{11}\left[\hat{A}-V_{12} V_{22} \hat{C}\right]^{T}+\left[\hat{A}-V_{12} V_{22} \hat{C}\right] X_{11} \\
& -X_{11}\left[\hat{C}^{T} V_{22}^{-1} \hat{C}\right] X_{11}+\left[V_{11}-V_{12} V_{22}^{-\ell} V_{12}^{T}\right]=0 .
\end{aligned}
$$

Using the solution $\left[X_{11}\right]$ to determine the estimator matrix:

$$
L=\left(X_{11} \hat{C}^{T}+V_{12}\right) V_{22}^{-1},
$$

the resulting optimal state estimator equation is

$$
\dot{\hat{q}}(t)=[\dot{A}+\dot{B} G-L \dot{C}-L \dot{D} G] \hat{q}(t)+L[\vec{z}(t)],
$$

where the total feedback gain matrix is defined as:

$$
G \doteq\left[B_{r}^{+}\left[G_{1} G_{2}\right] H_{m}+\left[I-B_{r}^{+} B_{r}\right] G_{z}\right] .
$$

Since a portion of the gain matrix must satisfy the conditions for modal space control, an output feedback problem can be constructed by yet another augmentation of the states to guarantee that $r$ modes will be notched in an orthogonal manner.

Define the new state and noise vectors as:

$$
\vec{x}(t) \doteq\left\{\begin{array}{l}
\vec{q}(t) \\
\dot{q}(t)
\end{array}\right\} \quad \vec{\mu}(t) \doteq\left\{\begin{array}{l}
\vec{w}(t) \\
\vec{v}(t)
\end{array}\right\}
$$

and the matrices:

$$
\begin{aligned}
G_{m} & \doteq B_{r}^{+}\left[\begin{array}{ll}
G_{1} & G_{2}
\end{array}\right] H_{m} \\
M_{z} & \doteq\left[I-B_{r}^{+} B_{r}\right] \\
\bar{A} & \doteq\left[\begin{array}{cc}
\hat{A} & \hat{B} G_{m} \\
L \hat{C} & {\left[\hat{A}+\hat{B} G_{m}-L \hat{C}\right]}
\end{array}\right] \\
\bar{B} & \doteq\left[\begin{array}{c}
\hat{B} M_{z} \\
\hat{B} M_{z}
\end{array}\right] \\
\bar{D} & \doteq\left[\begin{array}{cc}
\hat{E} & 0 \\
L \hat{D} \hat{F} & L
\end{array}\right]
\end{aligned}
$$

then the equivalent output feedback problem is:

$$
\begin{aligned}
\dot{\vec{x}}(t)= & \bar{A} \vec{x}(t)+\bar{B} u_{z}(t)+\bar{D} \vec{\mu}(t) \\
& ; u_{z}(t) \doteq G_{z}\left[\begin{array}{ll}
0 & I
\end{array}\right] \vec{x}(t) \\
y(t)= & {\left[\begin{array}{ll}
C & 0
\end{array}\right] \vec{x}(t) . }
\end{aligned}
$$

It is clear that $\left[G_{z}\right]$ is the control gain matrix which must be utilized for additional control objectives beyond modal space control. $\left[G_{z}\right]$ can be found optimally by satisfying constraint equations for a typical output feedback problem as shown in [18].

\section{Numerical Example}

Simulations of the Notch Control concept are performed on the ASTREX FEM model. Six modes are retained using the modal cost analysis technique [19]. Notice in Figure 1 that the ASTREX test article has two linear thrusters in the primary structure which have control authority in the $z$-direction, one disturbance source (vibrating cryocooler) at the secoldary placed at a 45 degree angle in the $x-y$ plane, and twelve accelerometers in the $x$ - and $y$-directions at six nodes of the secondary supports.

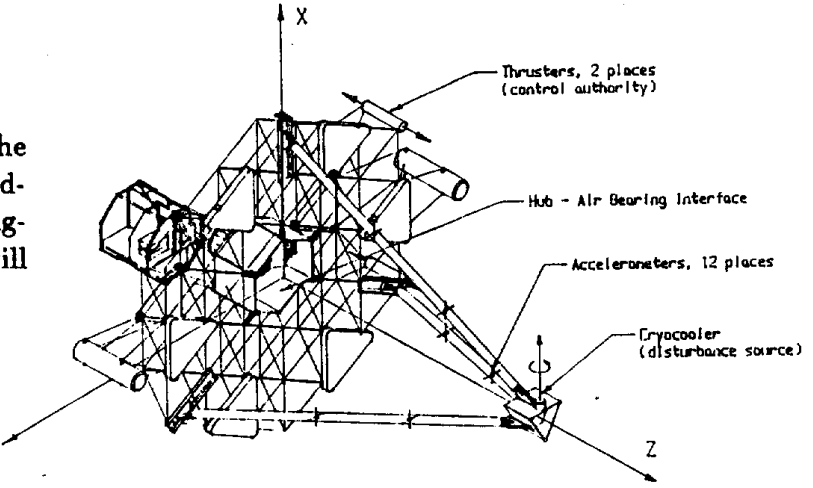

Figure 1: ASTREX Test Article

Figure 2 shows a superposition of a continous 11.2 $\mathrm{Hz}$ sinusoidal disturbance, which is close to the third modal frequency $(11.4 \mathrm{~Hz})$, and an impulse that occurs at $t=20$ seconds, both emanating from the cryocooler. Figure 3 is the resulting open loop res wase (displacement time history) in the $\mathbf{x}$ direction for one of the accelerometer locations. The control ohiort:-e is to attenuate the third mode while controlling the 

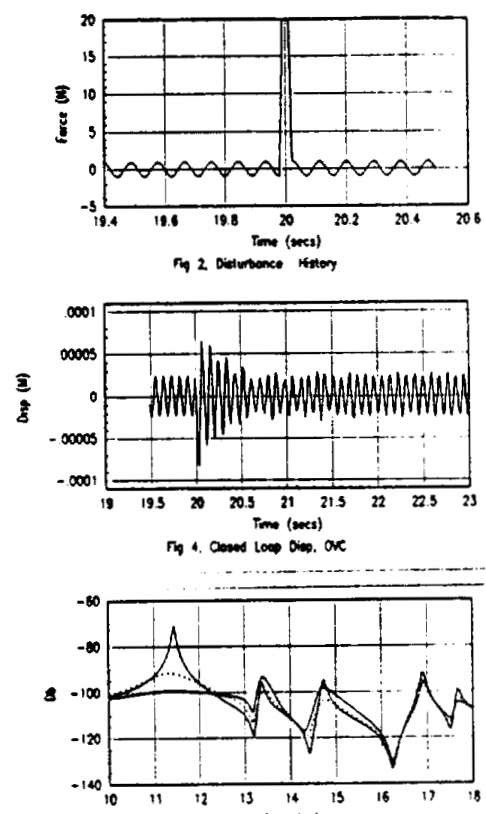

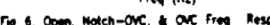

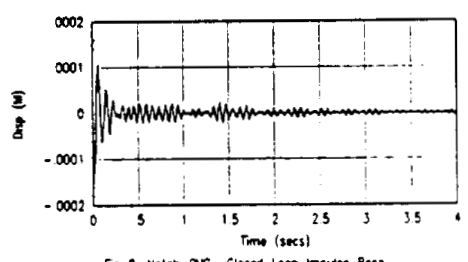

rig 8. Noten-mic closed loos impuse Ress

output variances due to a pulse excitation. To show the effectiveness of this control scheme, an OVC (Output Variance Constraint, [20]) controller was first synthesized with position and velocity constraints at the accelerometer location. The closed loop response to the disturbance displayed in Figure 2 is shown in Figure 4. In our example, the third mode is significantly effected by excitation from the cryocooler, which justifies increasing the damping ratio of that mode by modal notching. By choosing the damping ratio of the third mode to be 0.6 , the disturbance rejection using modal notch control is presented in Figure 5. The comparison of frequency responses of the OVC and modal notch controlled systems with the uncontrolled open loop system is shown in Figure 6, and clearly shows the significance of using modal notch control to eliminate key modes while simultaneously meeting output variance requirements. Figures 7,8 ,
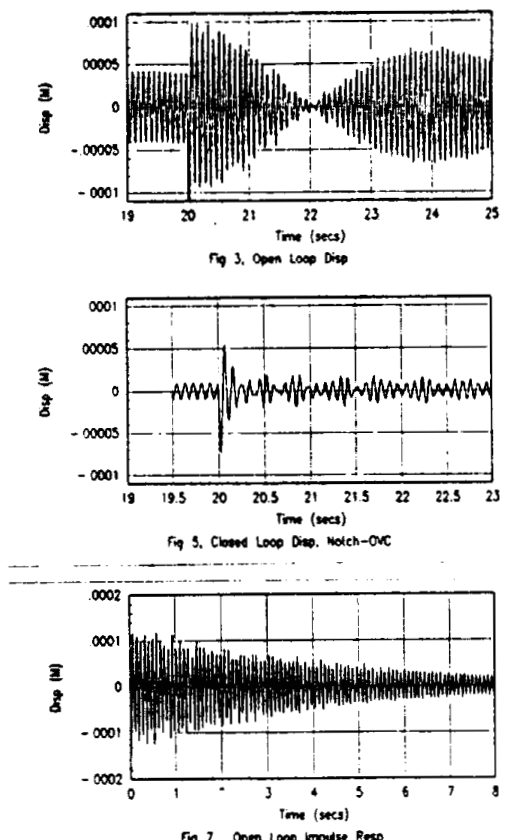

fro 7, open ioop unouse pesp

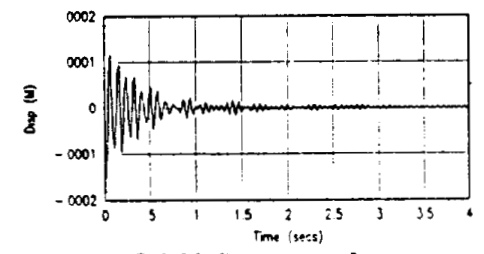

Fing, ove closed loop mputse Resp

and 9 are plots of the open loop, modal notch using OVC, and the OVC impulse responses with which the latter two have identical output variances using the two control techniques.

\section{Conclusions}

An analytical technique was presented to control the response of a dynamical system exposed to frequency dependent and random disturbances. The method is advantageous because multiple control schemes can be combined to achieve disturbance rejection which results in a useful approach for solving practical controls problems. By supplementing a control scheme with an orthogonal, 1MSC-based control scheme, one can readily devote energy to the damping of particularly troublesom: :-.udes to overcome frequency dependent disturbances. This is a practical approach 
when one considers that a structure's frequency dependent disturbances are not always predictable, and due to the addition of hardware, will most likely change during the service life of the structure.

\section{References}

[1] Ross, R.G.,Requirements for Longlife Mechanical Cryocoolers for Space Application, Cryogenics, pp. 233-238, Vol. 30, 1990.

[2] Meirovitch, L. and Baruh, H., A Comparison of Control Techniques for Large Flexible Systems, J. Guidance, Control, and Dynamics, pp. 302310, Vol. 6, No. 4, Jul-Aug, 1983.

[3] Meirovitch, L., and Baruh, H., Robustness of the Independent Modal Space Control Method, J. Guidance, Control, and Dynamics, pp. 2025, Vol. 6, No. 1, 1983.

[4] Oz, H. and Meirovitch, L.,Optimal Modal Space Control of Flexible Gyroscopic Systems, J. Guidance, Control, and Dynamics, pp. 218-226, Vol. 3, No. 3, 1980.

[5] Bennighof, J.K. and Meirovitch, L., Active Suppression of Traveling Waves in Structures, J. Guidance, Control, and Dynamics, pp. 555-567, Vol. 12, No. 4, July-Aug. 1989.

[6] Meirovitch, L. and Baruh, H. The Implementation of Modal Filters for Control of Strursures, J. Guidance, Control, and Dynamics, pp. 107116, Vol. 8, 1985.

[7] Sinha, A. and C.K. Kao Independent Modal Sliding Mode Control of Vibrations in Flexible Structures, J. Sound and Vibration, pp. 352$358,147(2), 1991$.

[8] Lindberg, R.E. and Longman, R.W., On the Number and Placement of Actuators for Independent Modal Space Control, Paper No. 82 1435, AIAA/AAS Astrodynamics Conference, San Diego, CA, Aug. 9-11,1982.

[9] Baz, A. and Poh, S., Experimental Impicirs ...itation of the Modified Independent Modal Space Control Method J. Sound and Vibration, pp. 133-149, 139(1), 1990.
[10] Silverberg, L.M., and Meirovitch, L.,BlockIndependent Control of Distributed Structures, Optimal Control, Applications and Methods, pp. 281-289, Vol. 6, No. 3, July-Sept 1985.

[11] Nguyen, C.C., Implementation of Actuators for the Independent Modal Space Control Scheme, Computers Elec. Engng., pp. 75-90, Vol. 17, No. 2, 1991.

[12] Berg, J.L., Identification and Control of Lightly Damped, Large Space Structures: An Experimental Evaluation, PhD Dissertation, Virginia Polytechnic Institute \& State Universily, July 1992.

[13] Haddad, W.M. and Bernstein, D.S., Controller Design with Regional Pole Constraints, IEEE Trans. Automat. Contr., Vol37, No. 1, January 1992.

[14] Lu, J., Chiang, H. and Thorp, S., Partial Eigenstructure Assignment and its Application to Large Scale Systems, IEEE Trans. Automat. Contr., Vol 36, No. 3, March 1991.

[15] Rao, C.R. and Mitra, S.K., Generalized Inverses of Matrices and it's Applications, John Wiley \& Sons, 1971.

[16] Kwakernaak, H. and Sivan, R., Linear Optimal Control Systems, John Wiley \& Sons, Inc., 1972.

[17] Hu, A., Skelton, R.E., Norris, G.A. and Cossey, D., Selection of Sensors and Actuators with Applications to the Astrex Facility, $4^{\text {th }}$ NASA/DOD CSI Conference, Orlando, Fl. Nov. 1990.

[18] Skelton, R.E., Dynamic Systems Control, Linear Systems Analysis and Synthesis, John Wiley \& Sons, Inc., 1990.

[19] Skelton, R.E., Singh, R. and Ramakrishnan, J., Component Model Reduction by Component Cost Analysis, AIAA Guidance and Controls Conf., Minneapolis, Minn., 1988.

[20] Hsieh, C., Skelton, R.E. and Damra, F.M., Minimum Energy Controllers with Inequality Constraints on Output Variances, Optimal Control Applications and Methods, Vol 10, PP 347-366 (1989) 